\title{
Designing Personalisation in a Environmental Recommendation System: Differences of AHP and Fuzzy AHP
}

\author{
Katerina KABASSI \\ Department of Environment, Ionian University
}

\begin{abstract}
Different methods have been proposed for designing the personalization process in a recommendation system. In the past, multi-criteria decision making theories have been proposed for the design of stereotypes in a recommendation system for environmental awareness. The main objective of this paper is on presenting the main differences when applying the fuzzy AHP and AHP for designing the weights of criteria in a recommendation system that its personalization process is based on multi-criteria decision making theories.
\end{abstract}

Keywords. Multi-Criteria Decision Making, Personalisation, Recommendation Systems, Stereotypes

\section{Introduction}

The research discipline of recommender systems arose to address the problem of information or choice over-abundance, i.e., to help users find information or items that are most likely to be interesting to them or to be relevant to their needs [1]. Recommendation systems have been expanded for use in mobile phones since these devices have been widely spread. Especially, Smartphone crowdsourcing enables realtime data gathering and gives devices access to a wealth of information about each device's user. As a result, recommendation systems for mobile phones have been developed. The main advantage of those systems is that they can make use of the large number of modalities for interaction between them and their environment [2]. Implicit information coming from Smartphone crowdsourcing can be combined with location and time specific data can further utilize software adaptation [8].

Using all these information, we developed a mobile recommendation system for providing personalized information about Centuries-Old Olive Groves. The system is called OldGrove and is aimed to be used in the Ionian Islands. In order to provide personalized interaction, OldGrove employs user modeling techniques. More specifically, the system uses multi-criteria decision making techniques (MCDM) in combination with individual user modeling and stereotypes for providing personalized interaction. Stereotypes are used for providing the weights of the criteria and individual user modeling the values of the criteria. This paper aims at presenting the design of stereotypes using Analytic Hierarchy Process (AHP) [10]. The main objective of this research is to present a comparative analysis AHP and Fuzzy AHP (FAHP) [3] for designing stereotypes in a recommendation system. Comparison of AHP and FAHP has been implemented before but in completely different context $[3,6,7]$. 


\section{Designing the Personalisation in a Recommendation system}

Personalised interaction may be provided when the system maintains user models. A user model is a collection of information on the knowledge, interests and other characteristics of each user. Stereotypes [9] are used in user modeling in order to provide default assumptions about individual users belonging to the same category according to a generic classification of users that has previously taken place. This method has the advantage of providing personalized recommendations from the first interaction of the user with the system.

In this approach, the system uses a combination of stereotypes and individual user modeling. For the personalization of the recommendations, personalization uses multicriteria decision making techniques for combining the data about the user that are stored in the user model. Taking into account the methods of personalization as well the multi-criteria decision making techniques used, the system uses stereotypes that provides the weights of the criteria and individual user model that provides the values of the criteria.The individual user model and the activated stereotype are loaded during the first phase of the recommendation mechanism, which is called cold start mechanism. Figure 1 presents all the steps the system implements for providing personalized recommendations.

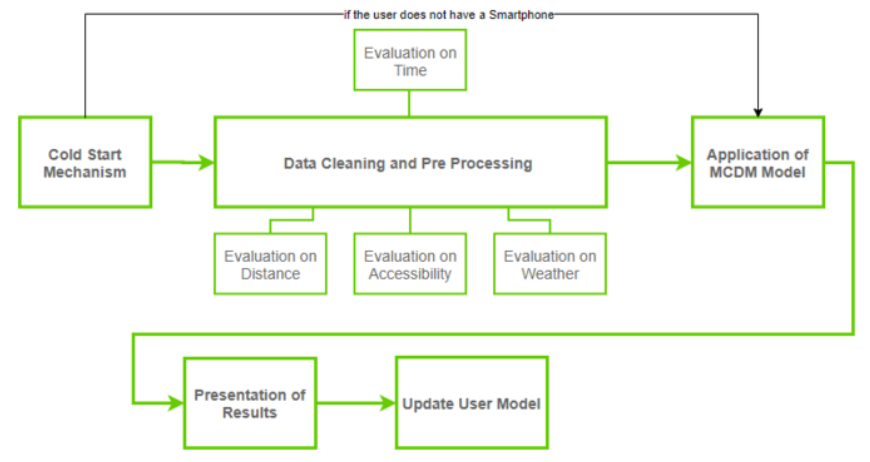

Figure 1. The mechanism of personalized recommendation of OldGrove

In the cold start mechanism, all possible recommendations are generated. If the system tracks a Smartphone, then the module 'Data Cleaning and Pre-Processing' runs. Otherwise, the system evaluates all recommendation that have been generated using the MCDM model. The 'Data Cleaning and Pre-Processing' module aims at reducing the possible recommendations before these are evaluated by the application of the MCDM model. The 'Data Cleaning and Pre-Processing' module uses criteria such as Distance, Accessibility, Weather and Time.

The MCDM model uses other four criteria for evaluating recommendations:

- Knowledge: Compatibility with the user's knowledge is also considered very important while evaluating the different pieces of information.

- Interests: Compatibility with user's interests is considered important in order to select the most interesting pieces of information for each user

- Skills: Compatibility with user's skills is considered important so that the users could comprehend the information provided to them.

- Learning Needs: Compatibility with the learning needs of a user could also be use for evaluating different pieces of information. 
These criteria are not considered as equally important by the system. The values of the weights of importance are acquired by the stereotype that has been activated during the cold start mechanism.

\section{Stereotype Design: Fuzzy AHP vs AHP}

In the early stages of the software's life cycle in order to find out the possible categories of users, we conducted an expert-based study [4], which revealed that the stereotypes that could be used for categorizing the possible users of the system are: Foresters, Agriculturalists, Historians, Farmers, Tourists, Residents.

AHP is a very well known MCDM theory. Its main advantage is that it presents a formal way of quantifying the qualitative criteria. A main reason for selecting AHP over other MCDM theories is that it presents a well defined way for calculating the weights of the criteria while most of MCDM theories have only defined the way criteria and weights are combined to rank alternatives but consider the weights of the criteria as already known.

Table 1. The weights of the criteria in the different stereotypes using AHP

\begin{tabular}{lllll}
\hline & Knowledge & Interests & Skills & Learning Needs \\
\hline Foresters & 0.566 & 0.153 & 0.233 & 0.049 \\
Agriculturalists & 0.541 & 0.091 & 0.227 & 0.141 \\
Historians & 0.574 & 0.235 & 0.235 & 0.055 \\
Farmers & 0.473 & 0.081 & 0.302 & 0.144 \\
Tourists & 0.363 & 0.387 & 0.196 & 0.054 \\
Residents & 0.418 & 0.143 & 0.346 & 0.093 \\
\hline
\end{tabular}

Table 2. The weights of the criteria in the different stereotypes using FAHP

\begin{tabular}{lllll}
\hline & Knowledge & Interests & Skills & Learning Needs \\
\hline Foresters & 0.556 & 0.166 & 0.229 & 0.049 \\
Agriculturalists & 0.542 & 0.118 & 0.212 & 0.128 \\
Historians & 0.569 & 0.153 & 0.224 & 0.054 \\
Farmers & 0.417 & 0.136 & 0.308 & 0.139 \\
Tourists & 0.355 & 0.421 & 0.175 & 0.049 \\
Residents & 0.374 & 0.147 & 0.334 & 0.146 \\
\hline
\end{tabular}

We have used AHP to execute three steps: (1) structuring the hierarchy of criteria; (2) assessing the expert evaluations by pair-wise comparison of the criteria; and (3) using the eigenvector method to yield weights for the four criteria. Through AHP, the importance of several criteria is obtained from a paired comparison process, in which the relevance of attributes or categories of drivers of intangible assets are matched twoby-two in a hierarchical structure [3]. The application of AHP revealed the weights of criteria for each stereotype of users (Table 1).

Except for AHP, fuzzy AHP has also been extensively used. Fuzzy AHP is based on the fuzzy interval arithmetic with triangular fuzzy numbers and confidence index $\alpha$ with an interval mean approach has been applied to weight evaluative element [3]. The main difference of AHP with FAHP is that the latter uses linguistic terms that are associated with triangular fuzzy numbers. A detailed application of FAHP for the estimation of weights for the different categories of users are presented in detail in [4] and are presented in summary in Table 2.

One can easily observe that the two models provide similar results but not identical. Very similar were the results for foresters, whereas main differences are presented in all the other stereotypes. In the Agriculturalists, Historians, Farmers, and Tourists the 
main difference is observed in the weights of the criterion Interests. For example, Interests are considered to be more important for agriculturalists when weights are calculated with AHP than when are calculated with FAHP. As far as the stereotype Residents is concerned differences are observed in the weights of criteria Knowledge and Learning Needs. Testing 5 scenarios where the values of the criteria were known, revealed that the differences were minor and did not affect the results of the recommendation system.

\section{Conclusions}

In this paper we have described the design of stereotypes in a recommendation system for environmental information. For the design of the stereotypes, we have used in turn AHP and FAHP. The main focus of the paper is on presenting the differences that occur when applying both models. Small differences occurred in the weights of the criteria but the testing of the both sets by using 5 specific scenarios revealed that the differences were not important. FAHP proved to be easier for expert users to implement. Similarly to [5], it is also demonstrated the advantage of being able to capture the vagueness of human thinking and to aid in solving the research problem through a structured manner and a simple process. It is among our future plans to implement the system with both sets of weights for each stereotype and test its effectiveness with real users.

\section{References}

[1] G. Adomavicius, Y. Kwon, Multi-criteria recommender systems, Recommender Systems Handbook, (2015), 847-880. http://dx.doi.org/10.1007/978-1-4899-7637-6_25.

[2] P. Bhargava, A. K. Agrawala, Modeling Users' Behavior from Large Scale Smartphone Data Collection, EAI Endorsed Trans. Context-aware Systems \& Applications, 3(10) (2016) https://doi.org/10.4108/eai.12-9-2016.151677

[3] Y. C. Chou, H. Y. Yen, C. C. Sun, J. S. Hon, Comparison of AHP and fuzzy AHP methods for human resources in science technology (HRST) performance index selection, 2013 IEEE International Conference on Industrial Engineering and Engineering Management, 2013, 792-796, doi: 10.1109/IEEM.2013.6962520.

[4] K. Kabassi, A. Martinis, Studies for Designing Stereotypes in a Mobile Recommendation System for Environmental Awareness. 15th IEEE International Workshop on Semantic and Social Media Adaptation \& Personalization (2020).

[5] G. Kabir, M.A.A. Hasin, Comparative Analysis of AHP and Fuzzy AHP Models for Multicriteria Inventory Classification. International Journal of Fuzzy Logic Systems (IJFLS), 1(1), 1-16.

[6] R. Mosadeghi, J. Warnken, R. Tomlinson, H. Mirfenderesk (2015) Comparison of Fuzzy-AHP and AHP in a spatial multi-criteria decision making model for urban land-use planning, Computers, Environment and Urban Systems, 49 (2015), 54-65,https://doi.org/10.1016/j.compenvurbsys.2014.10.001.

[7] M. Naderzadeh, H. Arabalibeik, M. R. Monazza, I. Ghasemi, Comparative Analysis of AHP-TOPSIS and Fuzzy AHP Models in Selecting Appropriate Nanocomposites for Environmental Noise Barrier Applications, Fluctuation and Noise Letters, 16(4) (2017) 1750038

[8] A. M. Otebolaku, M. T. Andrade, User context recognition using smartphone sensors and classification models, Journal of Network and Computer Applications, $66 \quad$ (2016), 3351https://doi.org/10.1016/j.jnca.2016.03.013G.

[9] E. Rich, Users are individuals: individualizing user models, International Journal of Human-Computer Studies, 51 (1999), 323-338.

[10] T. Saaty, G. Hu, Ranking by Eigenvector Versus Other Methods in the Analytic Hierarchy Process. Applied Mathematics Letters, 11(4) (1998), 121-125. 